\title{
On the Effective Elastic Properties of Macroscopically Isotropic Media Containing Randomly Dispersed Spherical Particles
}

D. Cojocaru

University of Delaware

Anette M. Karlsson

Cleveland State University, a.karlsson@csuohio.edu

Follow this and additional works at: https://engagedscholarship.csuohio.edu/enme_facpub

Part of the Mechanical Engineering Commons

How does access to this work benefit you? Let us know!

\section{Original Citation}

Cojocaru, D., and Karlsson, A. M., 2010, "On the Effective Elastic Properties of Macroscopically Isotropic Media Containing Randomly Dispersed Spherical Particles," Journal of Engineering Materials and Technology, 132(2) pp. 021011.

This Article is brought to you for free and open access by the Mechanical Engineering Department at EngagedScholarship@CSU. It has been accepted for inclusion in Mechanical Engineering Faculty Publications by an authorized administrator of EngagedScholarship@CSU. For more information, please contact library.es@csuohio.edu. 


\section{On the Effective Elastic Properties of Macroscopically Isotropic Media Containing Randomly Dispersed Spherical Particles}

A. M. Karlsson ${ }^{1}$ e-mail: karlsson@udel.edu

Department of Mechanical Engineering, University of Delaware, Newark, DE 19716
A computational scheme for estimating the effective elastic properties of a particle reinforced matrix is investigated. The randomly distributed same-sized spherical particles are assumed to result in a composite material that is macroscopically isotropic. The scheme results in a computational efficient method to establish the correct bulk and shear moduli by representing the three-dimensional $(3 D)$ structure in a two-dimensional configuration. To this end, the statistically equivalent area fraction is defined in this work, which depends on two parameters: the particle volume fraction and the number of particles in the $3 D$ volume element. We suggest that using the statistically equivalent area fraction, introduced and defined in this work, is an efficient way to obtain the effective elastic properties of an isotropic media containing randomly dispersed same-size spherical particles.

Keywords: particle reinforced composites, effective properties, finite element method

\section{Introduction}

Particle reinforced composites are a common form of heterogeneous material systems used in engineering structures. Significant efforts have been directed toward estimating the effective (i.e., macroscopic) properties of such multiphase materials based on the properties of the individual phases and the internal architecture of the microstructure. Reviews of both analytical and numerical prediction methods are given for example in Refs. [1-5]. Compared with real life testing, utilizing numerical methods provide a costeffective method for evaluating how changes in microstructural features affect the macroscopic properties of composite materials. Schemes employing the finite element method (FEM) are the most popular approach to model material samples containing details about the internal microstructural architecture.

To account for the random distribution of the particles in the matrix, a sufficient number of configurations must be generated to obtain an insight into the statistical distribution of the effective properties [6]. Even though computational power constantly increases, computing the effective properties for a random threedimensional (3D) structure is both cumbersome and time consuming to perform. Not only is the time to generate a 3D mesh significantly more time consuming than a two-dimensional (2D) mesh but a 3D mesh is intrinsically larger than a 2D mesh; i.e., it requires more nodes and therefore results in more degrees of freedom (DOF). Thus, a 3D mesh can become prohibiting since the solution time of the FE-simulations depends directly on the number of DOF in the model. In general, the computational time for most popular direct FE solvers is approximately proportional to the cube of the number of DOF (the $\mathrm{DOF}^{3}$ ). This estimate comes from the so-called Cholesky decomposition-the most time consuming operation of the solver-which shows that the decomposition-time of a symmetric, positive-definite matrix is governed by $n^{3}$, where $n$ is the dimension of the matrix. The actual time depends on the actual solver (i.e., algorithm) used to

\footnotetext{
${ }^{1}$ Corresponding author.
}

solve the FE equations. Consequently, it follows that 3D samples are considerably more expensive, approximately a factor of 1000 . It is therefore common to represent the $3 \mathrm{D}$ structure with a $2 \mathrm{D}$ structure.

However, it is not obvious how a 3D structure of spheres can be represented with a $2 \mathrm{D}$ simplification. For example, representing a $3 \mathrm{D}$ matrix reinforced with spherical participles in a $2 \mathrm{D}$ approximation results in that the 2D model may actually represent cylinders (fibers) instead of spheres if a plane strain approximation is used. Moreover, when the particles are randomly distributed, an arbitrary cut through the (3D) matrix will not result in (2D) circular particles that are of uniform (same-size) diameters.

The purpose of this work is to investigate how a 3D structure with randomly distributed same-sized particles can be represented with a 2D-FEM model when establishing the effective elastic properties. To this end, we will in introduce (in Sec. 3) the concept of the statistically equivalent area fraction $\bar{A}_{\text {fp }}$. In Sec. 4 we will show that $\bar{A}_{\text {fp }}$ can be used when reducing a $3 \mathrm{D}$-representative volume element (RVE) of a matrix reinforced with same-sized spherical particles to an equivalent $2 \mathrm{D}$ representative area. Before discussing the concept of the equivalent area fraction, we will briefly describe how the random samples were generated in the numerical simulations. The results are compared with well-known analytical approaches, including the Hashin-Shtrikman (HS) upper and lower bounds [7] and the self-consistent method [8]. A range of work shows that experimentally obtained data fall within these estimates, e.g., Refs. [9-13]. We will, in future studies, utilize the method of statistically equivalent area fraction when investigating more sophisticated structures, such as coated particles and damaged structures, with nonlinear and nonconservative response, where traditional analytical methods may not be applicable.

\section{Generating 3D Samples}

For same-size spheres, the theoretically, largest volume fraction of particles is 0.74048 for periodic packing (according to Kepler's onjecture [14]) and about 0.65 for random packing [15]. There re several approaches to generate a random distribution of pheres in a volume, for example, the random sequential adsorp- 
tion (RSA) [16] and the algorithm by Metropolis et al. [17]. The algorithm by Metropolis et al. [17] can reach the highest level of random packing since the algorithm starts with an ordered arrangement of particles (e.g., face centered cubic (FCC) lattice) and then randomly (using a Monte-Carlo approach) move each particle to a new position. However, to reach a true random distribution, with no trace of the original ordered structure, the algorithm theoretically requires infinitely many random moves. In computer simulations, this corresponds to a very large number of computations, and this is therefore a time consuming algorithm. In contrast, the RSA is an algorithm where randomly generated, nonintersecting spheres of same-size radius are sequentially inserted into the cube representing the 3D domain investigated. This method is associated with a "jamming limit," which is reached as the domain runs out of free space to place an additional sphere. Via computer simulations, Talbot and Schaaf [18] found that the "jamming volume fraction" is 0.382 . At this volume fraction, the nearest-neighbor distance $H_{1}$ is approximately $1.03 d$, where $d$ is the diameter of the particles [19]. That is, if the diameter of the same-size particles is of unit size, the smallest gap between the particles is on average 0.03 . Thus, the jamming volume fraction corresponds to a relatively high practical volume fraction for most real particle reinforced materials. We note here that particle volume fractions reported in the literature that are higher than about 0.35 are almost always nonspherical particles or spherical particles with a broad range of sizes [20].

In this work, we implemented an approach based on the RSA since this is a relatively fast and simple method and since it represents a broad spectrum of manmade composites. Similar approaches have been employed by other researchers (e.g., Refs. $[21,22])$. Our adoption of the RSA will be discussed next for clarity of the ensuing discussion.

Consider the cubical 3D specimen with sides $h$. Define the particle volume fraction $V_{\mathrm{fp}}$ by $V_{\mathrm{fp}}=\frac{4}{3} \pi r^{3} N / h^{3}$, where $r$ is the radius of the particles and $N$ is the number of spheres in the specimen. The procedure for generating random specimen configurations has been implemented using the PYTHON language [23,24]. The Mersenne Twister algorithm (which has a period of $2^{19,937}-1$ ) is used for random generation [25]. This algorithm is included in PYTHON [23]. We employ a Monte-Carlo approach to generate 3D samples with random particle distribution, where we consider the volume fraction $V_{\mathrm{fp}}$ and the number of particles in the specimen $N$ as parameters. The algorithm used is based on the following scheme. First, for each particle volume fraction considered, the radius of the particles $r_{\text {ref }}$ is established assuming a single sphere $(N=1)$ in a cube of unit volume $(h=1)$

$$
r_{\text {ref }}=\left(\frac{3 V_{\mathrm{fp}}}{4 \pi}\right)^{1 / 3}
$$

Next, for each particle volume fraction, the radius $r_{\text {ref }}$ is kept constant, while the $3 \mathrm{D}$ specimen is enlarged in order to include more inclusions. This approach is referred to as the successive domain enlargement and is commonly used, e.g., Refs. $[5,26]$. The size of the specimen is characterized by the length of each side in the cubic $3 \mathrm{D}$ domain $h_{\mathrm{RVE}}$

$$
h_{\mathrm{RVE}}=r_{\text {ref }}\left(\frac{4 \pi N}{3 V_{\mathrm{fp}}}\right)^{1 / 3}
$$

The cubic RVE is considered centered at the origin. An iterative routine generates a valid random configuration containing the prescribed number of particles $N$.

Only cases where the spheres are completely inside the cube are considered, i.e., the cube faces do not intersect the particles. This simplification is a reasonable assumption for the volume fractions considered and eliminates complications related to splitting the spheres intersecting the domain boundaries while enforcing a given volume fraction.
To ensure that the spheres are completely inside the cubic RVE, the center coordinates $\left(x_{i}, y_{i}, z_{i}\right)$ for each particle $i$ are generated according to the uniform distribution

$$
\begin{aligned}
& x_{i}=U\left(-h_{\mathrm{RVE}} / 2+\varepsilon_{h}+r_{\mathrm{ref}}, h_{\mathrm{RVE}} / 2-\varepsilon_{h}-r_{\mathrm{ref}}\right) \\
& y_{i}=U\left(-h_{\mathrm{RVE}} / 2+\varepsilon_{h}+r_{\mathrm{ref}}, h_{\mathrm{RVE}} / 2-\varepsilon_{h}-r_{\mathrm{ref}}\right) \\
& z_{i}=U\left(-h_{\mathrm{RVE}} / 2+\varepsilon_{h}+r_{\mathrm{ref}}, h_{\mathrm{RVE}} / 2-\varepsilon_{h}-r_{\mathrm{ref}}\right)
\end{aligned}
$$

Equation (3) also ensures that a space greater than $\varepsilon_{h}$ exists between the sphere and the cube walls. Next, the newly generated sphere is validated against the intersection with each of the previously generated spheres, by verifying that the distance between the sphere centers is greater than twice that of the particle radius,

$$
\sqrt{\left(x_{i}-x_{k}\right)^{2}+\left(y_{i}-y_{k}\right)^{2}+\left(z_{i}-z_{k}\right)^{2}}>2\left(r_{\mathrm{ref}}+\varepsilon_{h}\right)
$$

The inequality (4) is applied against any sphere $k$ already added to the specimen configuration and enforces that a minimum gap of $2 \varepsilon_{h}$ exists between neighboring spheres. Random generation of each sphere $i$ is attempted until either Eq. (4) is satisfied, or until a user-specified number of attempts per sphere $a_{i}$ is reached. If a valid sphere cannot be inserted after $a_{i}$ trials, the generation of the entire specimen is restarted. The procedure is repeated until all user-specified spherical particles have been successfully inserted in the cubic RVE and thus, a valid specimen has been obtained. The number of trials necessary to generate a valid specimen strongly depend on the volume fraction $V_{\mathrm{fp}}$. Segurado and Llorca [22] utilized the method discussed here for volume fraction up to $V_{\mathrm{fp}}=0.3$. Here, we limit the volume fractions to 0.275 to keep the computational time reasonable. ${ }^{1}$

\section{The Statistically Equivalent Area Fraction}

In this section, we introduce the concept of the statistically equivalent area fraction $\bar{A}_{\text {fp. }}$. In Sec. 4 we will show that $\bar{A}_{\text {fp }}$ can be used when reducing a $3 \mathrm{D}-\mathrm{RVE}$ of a matrix reinforced with spherical particles to an equivalent $2 \mathrm{D}$ representative area.

Based on the scheme presented in Sec. 2, a Monte-Carlo type approach is used to generate randomly dispersed spherical particles in a cubic (3D) specimen (Fig. 1). The spherical particles are assumed to have a constant (same-size) diameter. For simplicity, $\varepsilon_{h}=0$ in Eqs. (3) and (4). Each valid configuration generated by this routine is cross-sectioned by three orthogonal Cartesian planes $X Y(z=0), Y Z(x=0)$, and $Z X \quad(y=0)$ (Fig. 1). Each of these planes intersects some of the spherical particles in the cubic RVE (Fig. 1), forming a plane surface with disks (e.g., the cross section of a particle) and the matrix. For each random configuration of spheres $j$, the numbers of disks corresponding to each plane are denoted $N_{X Y}^{j}, N_{Y Z}^{j}$, and $N_{Z X}^{j}$. Furthermore, we define the equivalent area fraction as the total area of the disks resulting from the sphere-plane intersection, normalized with the area of the plane sections

\footnotetext{
${ }^{1}$ However, this method can be used effectively for area fractions up to $50 \%$ for $2 \mathrm{D}$ problems with circular inclusions (i.e., disks) being inserted into a square. We also note that $V_{\mathrm{fp}}=0.275$ leads to a nearest-neighbor distance of $H_{1}=1.06 d$ [19], which in practical terms is a relatively closed packed system.
} 


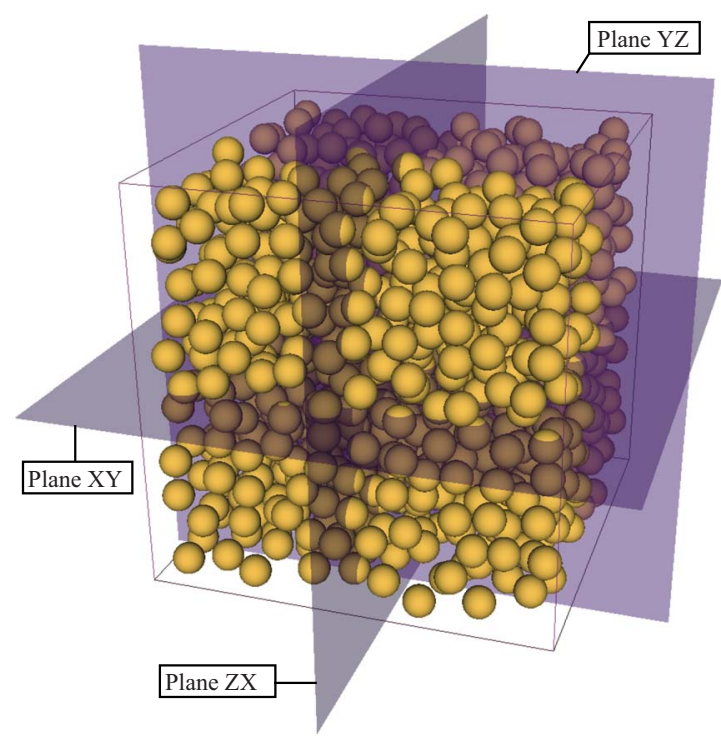

Fig. 1 A configuration with 1000 randomly generated nonintersecting spheres representing $25 \%$ volume fraction

$$
\begin{aligned}
& \left(A_{f, X Y}^{j}=\frac{1}{h_{\mathrm{RVE}}^{2}}\left(\sum_{\substack{i=1 \\
r_{x y, i}^{2} 0}}^{N} \pi r_{x y, i}^{2}\right) ; \quad r_{x y, i}^{2}=r_{\mathrm{ref}}^{2}-z_{i}^{2}\right. \\
& \left\{A_{f, Y Z}^{j}=\frac{1}{h_{\mathrm{RVE}}^{2}}\left(\sum_{\substack{i=1 \\
r_{y z, i}^{2} 0}}^{N} \pi r_{y z, i}^{2}\right) \pi r_{y z, i}^{2} ; r_{y z, i}^{2}=r_{\mathrm{ref}}^{2}-x_{i}^{2}\right. \\
& A_{f, \mathrm{ZX}}^{j}=\frac{1}{h_{\mathrm{RVE}}^{2}}\left(\sum_{\substack{i=1 \\
r_{z x, i}^{2} 0}}^{N} \pi r_{z x, i}^{2}\right) \pi r_{z x, i}^{2} ; r_{z x, i}^{2}=r_{\mathrm{ref}}^{2}-y_{i}^{2}
\end{aligned}
$$

Thus, the equivalent area fraction for each plane $A_{f, X Y}^{j}, A_{f, Y Z}^{j}$, and $A_{f, Z X}^{j}$, and the number of particles intersected by the three cutting planes $N_{X Y}^{j}, N_{Y Z}^{j}$, and $N_{Z X}^{j}$, respectively, are determined for each valid configuration $j=1,2, \ldots, C$, where $C$ is the number of configurations to be generated. $C$ was selected to be sufficient for convergence of the mean and standard deviation of the three equivalent area fractions. When a sufficient number of configurations are investigated, the equivalent area fraction for the orthogonal planes converges to one single value (Fig. 2). This implies that the morphology of the microstructure is isotropic, and consequently the elastic properties will be isotropic.

For a fixed volume fraction, an increasing number of particles (thus larger RVE) requires fewer configurations for the mean of the equivalent area fraction to converge (Figs. 2(a) and 2(b)). For a fixed number of particles, the convergence rate of the equivalent area fraction appears to be independent of the volume fractions (Figs. 2(c) and 2(d)). The equivalent area fraction assumes a Gaussian distribution (Fig. 3). The mean and standard deviation of the distribution depend on both the volume fraction and specimen size.

More insight to the convergence of the equivalent area fraction and the number of intersected spheres are obtained by investigating the mean and standard deviation for the distribution of these two quantities (Figs. 4 and 5, and tabulated in Table 1 for ease of use). Each point in Figs. 4 and 5 is obtained based on 50,000 randomly generated configurations. Interestingly, the mean of the area fraction changes nonmonotonically with increasing volume fraction, in particular for volume fractions higher than 20\% (Fig.
4) for samples containing 6-32 particles. Consequently, for simplicity we considered in the following only samples with a sufficiently high number of particles.

We define the statistically equivalent area fraction as the mean value of the equivalent area fraction $\bar{A}_{\text {fp }}$, and we denote the mean of the number of intersected particles $\bar{N}$. These quantities are summarized in Table 1. We will show in Sec. 4 that $\bar{A}_{\text {fp }}$ and $\bar{N}$ can be used for estimating the effective elastic properties of a 3D-RVE based on 2D-FE models with same-size particles (disks). Table 1 can be used for future reference to determine $\bar{A}_{\text {fp }}$ and $\bar{N}$. The statistically equivalent area fraction depends on the volume fraction $V_{\mathrm{fp}}$ and on the number of particles included in the specimen $N$.

\section{Effective Properties of a Particle Reinforced Medium}

In this section, we will investigate the effective shear and bulk moduli of a matrix reinforced with randomly distributed same-size spherical particles. We will show that a $2 \mathrm{D}$ simulation (employing same-sized 2D circular particles) can be used if the particle diameter is based on the statistically equivalent area fraction $\bar{A}_{\mathrm{fp}}$ (defined in Sec. 3).

To this end, we will adopt a numerical scheme presented by Zohdi and Wriggers [6], utilizing FEM, that predicts effective elastic properties in multiphase materials with a random dispersion of same-sized spherical particles. The idea is based on calculating the response for multiple configurations, where the particles are distributed randomly in the matrix in each simulation. Based on this, the mean and standard deviation for the bulk and shear moduli are determined. We extend the method presented by Zohdi and Wriggers [6] to investigate both two- and threedimensional-FE models of randomly distributed particles.

4.1 Definition of the Finite Element Models. The effective bulk and shear moduli for materials assumed macroscopically isotropic can be determined by using the commonly employed approach $[1,5,6]$

$$
\begin{gathered}
3 K_{a}=\frac{\left\langle\sigma_{k k} / 3\right\rangle_{\Omega}}{\left\langle{ }_{k k} / 3\right\rangle_{\Omega}} \\
2 G_{a}=\frac{\left\langle\sigma_{i j}^{\prime}\right\rangle_{\Omega}\left\langle\sigma_{i j}^{\prime}\right\rangle_{\Omega}}{\left\langle\begin{array}{c}
\prime \\
k l
\end{array}\right\rangle_{\Omega}\left\langle{ }_{k l}^{\prime}\right\rangle_{\Omega}}
\end{gathered}
$$

The summation convention is assumed in Eq. (6), and

$$
\langle\chi\rangle=\frac{1}{|\Omega|} \int_{\Omega} \chi d \Omega
$$

where $\Omega$ is the whole domain of the specimen investigated. In the context of the finite element method this integral can be seen as the sum of integrals over the deformed domain $\Omega^{e}$ of each element, i.e.,

$$
\int_{\Omega} \chi(\mathbf{x}) d \Omega=\sum_{e=1}^{N_{e l}} \int_{\Omega^{e}} \chi(\mathbf{x}) d \Omega^{e}
$$

where

$$
\Omega=\underset{e}{\cup} \Omega^{e}
$$

Using the mapping $\mathbf{x}=\mathbf{x}(\xi)$ from the deformed element domain $\Omega^{e}$ to the so-called parent element domain $\Omega_{\square}^{e}$, the integral becomes

$$
\int_{\Omega^{e}} \chi(\mathbf{x}) d \Omega^{e}=\int_{\Omega_{\square}^{e}} \chi(\mathbf{x}(\xi)) J^{\xi} d \Omega_{\square}^{e}
$$

where 


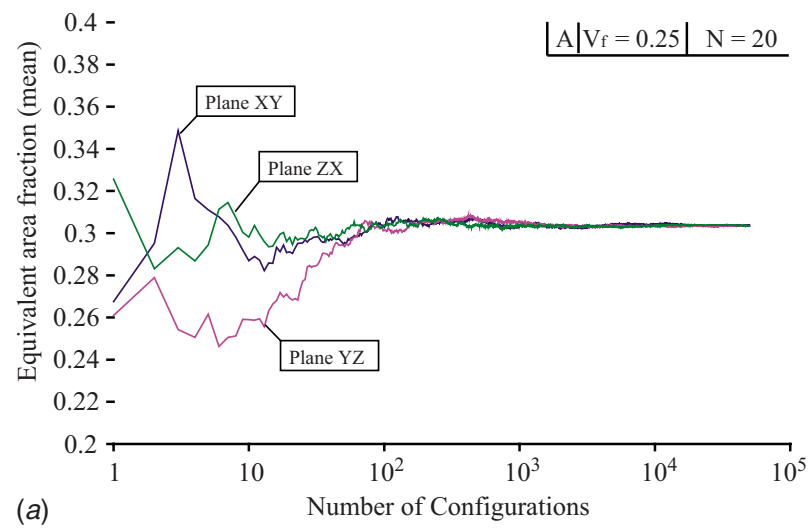

(a)

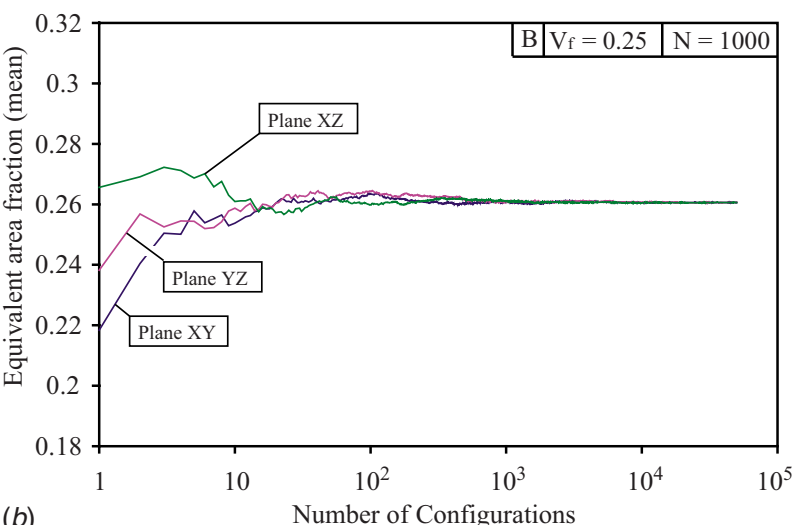

$(b)$
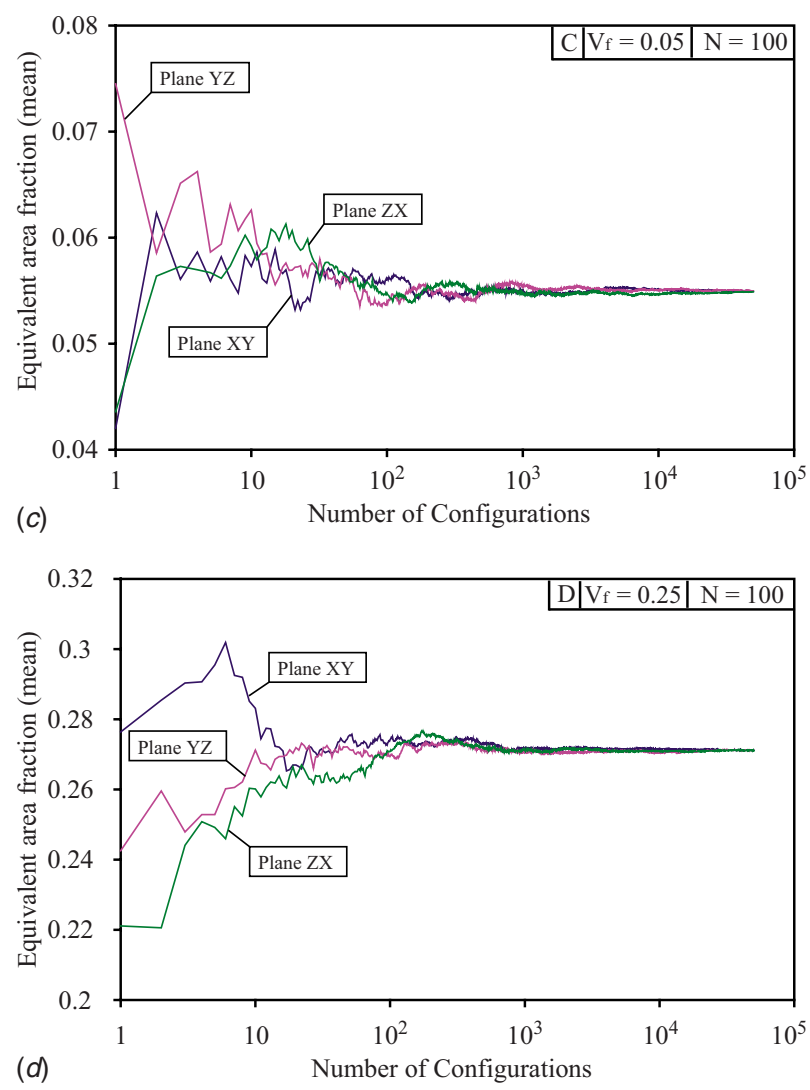

Fig. 2 Equivalent area fraction as a function of the number of configurations investigated, obtained by cross-sectioning the 3D-RVE simultaneously with three orthogonal planes for (a) $\mathbf{N}$ $=20$ particles and $V_{\mathrm{fp}}=\mathbf{0 . 2 5}$, (b) $N=1000$ particles and $V_{\mathrm{fp}}=\mathbf{0 . 2 5}$ (c) $N=100$ particles and $V_{\text {fp }}=0.05$, and (d) $N=100$ particles and $V_{\mathrm{fp}}=\mathbf{0 . 2 5}$
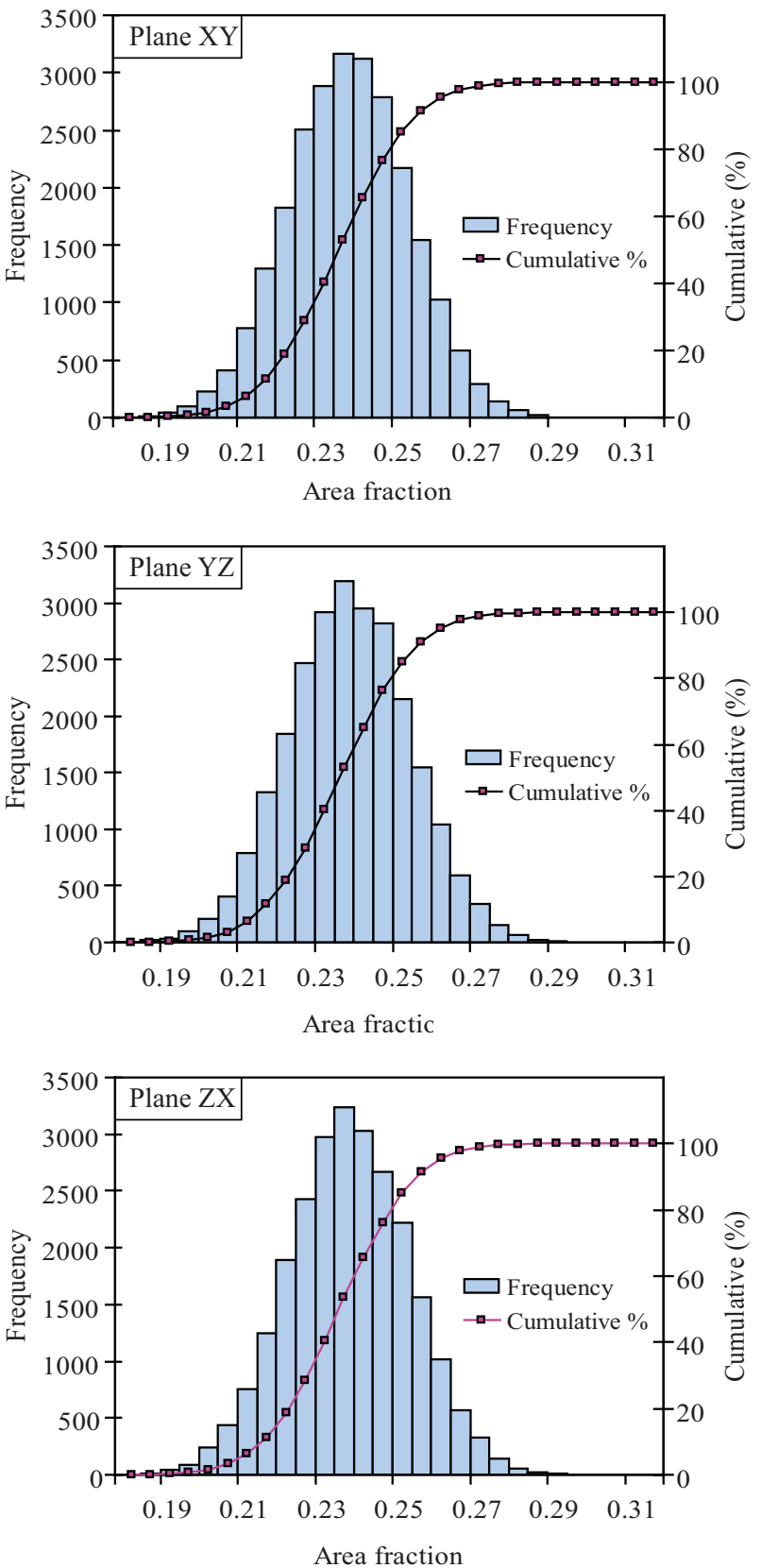

Fig. 3 Distribution of the equivalent area fraction obtained for $V_{f}=0.22$ and $N=1000$ particles by cross-sectioning the 3D cubic RVE with the three orthogonal planes. Histograms computed using 50,000 random configurations.

$$
J=\operatorname{det}\left(\left[\frac{\partial x_{i}(\boldsymbol{\xi})}{\partial_{j}}\right]\right)
$$

is the element Jacobian. Furthermore, the integral over the parent element domain can be approximated using a numerical quadrature formula. Gauss quadrature formulas are commonly used in the FEM and they are also employed in our implementation. Details on numerical integration in the context of the FEM can be found for example in Refs. [27-29]. Also, in Eq. (6) the deviatoric components are defined by $\sigma_{i j}^{\prime}=\sigma_{i j}-\frac{1}{3} \sigma_{k k} \delta_{i j}$ and ${ }_{i j}^{\prime}={ }_{i j}-\frac{1}{3}{ }_{k k} \delta_{i j}$, where $\sigma_{i j}$ denotes the components of the stress tensor where $\sigma_{k k}$ $=\sigma_{11}+\sigma_{22}+\sigma_{33}$ (thus $\sigma_{k k} / 3$ is the hydrostatic stress), $i j$ are the components of the strain tensor, where ${ }_{k k}={ }_{11}+{ }_{22}+33$ and $\delta_{i j}$ is the Kronecker delta ( $\delta_{i j}=1$ if $i=j$ and $\delta_{i j}=0$ otherwise). Equations (6) follow from Hooke's law for isotropic, linear elastic 

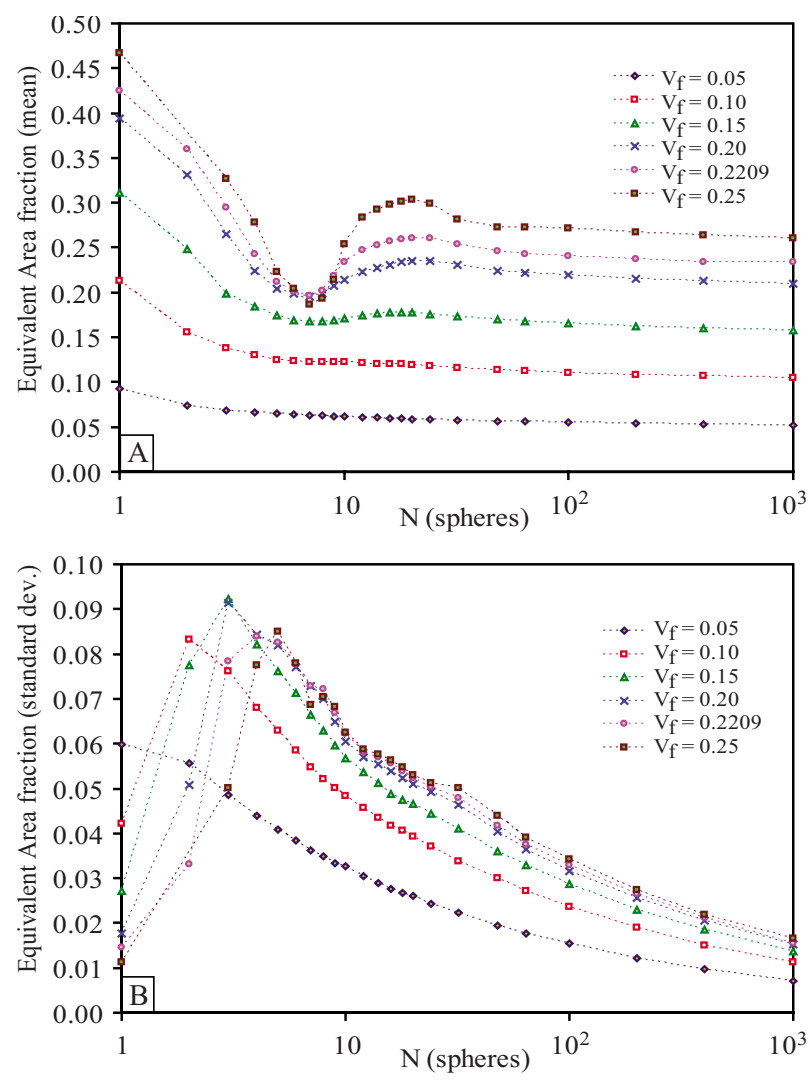

Fig. 4 Equivalent area fraction as the function of the number of particles $N$ obtained by cutting the cubic RVE with the $X Y$ plane, for various volume fractions: (a) the mean value and (b) the standard deviation. (Each point is based on 50,000 randomly generated configurations.)

materials, viz. $\left\langle\sigma_{i j}\right\rangle_{\Omega}=L_{i j k l}\left\langle{ }_{k l}\right\rangle_{\Omega}$, where $L_{i j k l}=K_{a} \delta_{i j} \delta_{k l}+G_{a}\left[\delta_{i k} \delta_{j l}\right.$ $\left.+\delta_{i l} \delta_{j k}-(2 / 3) \delta_{i j} \delta_{k l}\right]$ is the effective elasticity tensor.

In order to determine the elasticity tensor for the considered RVE, the RVE is typically subjected to selected boundary conditions so to relate stress to strain $[1,5,6]$. To this end, we use the prescribed boundary conditions $\left.u_{i}\right|_{\Omega}$, which are linear displacements given by (assuming summation convention)

$$
\left.u_{i}\right|_{\Omega}=k_{i j} x_{j}, \quad i, j=1, \ldots, N_{\mathrm{dim}}
$$

where $k_{i j}$ are constants and $N_{\mathrm{dim}}=2$ for 2D-FE models and $N_{\mathrm{dim}}$ $=3$ for $3 \mathrm{D}-\mathrm{FE}$ models. In the present study we used $k_{i j}=1$ $\times 10^{-3}$. Equation (7) offers a convenient way to create various deformation states. For example, in $2 \mathrm{D}$, if $k_{12}=0$, the model is subjected to biaxial displacements. The accurate estimation of effective shear modulus requires the off-diagonal terms to be nonzero whereas the effective bulk modulus requires nonzero diagonal terms [5]. These boundary conditions are applied in the commercial program ABAQUS using the user-subroutine DISP [30].

Two alternative approaches to formulate the mesh will be considered: (i) unaligned mesh or (ii) aligned mesh $[5,6,26]$. The terms aligned and unaligned refer to the orientation of the element faces (3D) or edges (2D) with respect to the interface between the matrix and the particle $[5,6,26]$. An aligned mesh implies that the interface geometry is modeled explicitly. A schematic of the two approaches is presented in Fig. 6 for a 2D model, but one can easily imagine the extension to 3D models.

Both approaches have advantages and disadvantages: The unaligned mesh (Fig. 6(a)) assumes a structured mesh that is easy to generate, since the RVE is usually cubic. However, the unaligned
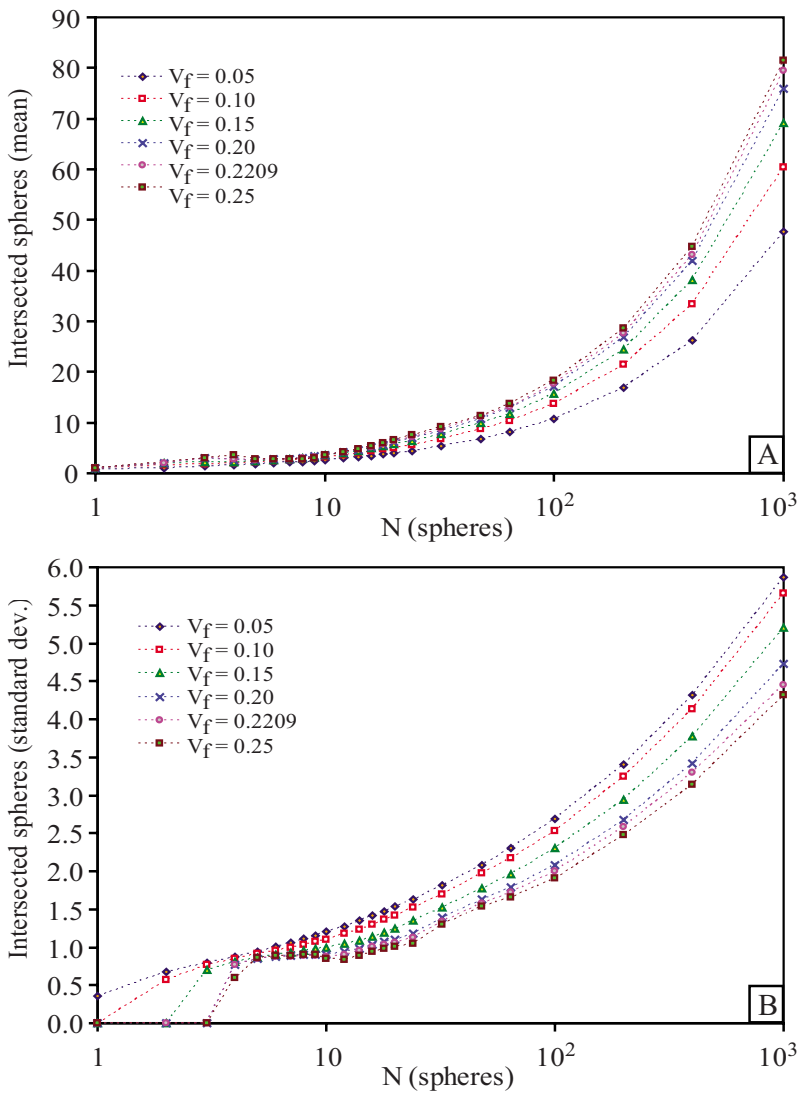

Fig. 5 Number of the particles intersected by plane $X Y$ as the function of the number of particles $\boldsymbol{N}$ for various volume fractions: (a) the mean value and (b) the standard deviation

mesh requires a large number of elements since the material interface is not modeled explicitly. This may lead to prohibitively large models for the available computational resources. ${ }^{2}$

In the aligned mesh, the interfaces can be modeled accurately, but generating an aligned mesh (Fig. 6(b)) is more computationally expensive. However, the resulting FE model is usually smaller than for an unaligned mesh since smaller elements can be used near the interfaces whereas larger elements can be used in regions within the bulk material. Thus, this approach is more cost-effective.

Zohdi and co-worker $[5,6,26]$ used an unaligned meshing approach to simulate 3D specimens. Here, we will extend their procedure for: (i) 2D models with aligned mesh and (ii) 3D models with both aligned and unaligned meshes. Trilinear hexahedral elements with a $2 \times 2 \times 2$ Gauss integration have been used by Zohdi and co-worker $[5,6,26]$ for the elements completely embedded in one of the material phases. Here, we use biquadratic serendipity ( 8 node) elements integrated by a $3 \times 3$ Gauss rule for the $2 \mathrm{D}$ models. For the 3D models, we use trilinear ( 8 node) elements for the unaligned meshes (with a $2 \times 2 \times 2$ integration rule) and quadratic (10 node) tetrahedral elements (with a four point integration rule) for the aligned meshes. These element formulations are available in the commercial software ABAQUS [31]. The generation of the geometry follows the description in Sec. 2, and we set ${ }_{h}$ to

\footnotetext{
${ }^{2}$ Special numerical integration rules might be used for the elements crossed by the theoretical interface between the materials to alleviate this problem. For example, a particular $5 \times 5 \times 5$, the integration rule was used by Zohdi and co-worker [5,6,26] for the elements crossed by the interface. However, the application of these higher order quadrature rules requires additional programming effort, and is not always possible to implement for a commercial FE software (depending on the software
} used). 
Table 1 The mean number of intersected particles and the statistically equivalent area fraction

\begin{tabular}{|c|c|c|c|c|c|c|c|c|c|c|c|c|}
\hline \multirow[b]{3}{*}{ No. of spheres } & \multicolumn{6}{|c|}{ Mean of intersected number of particles $\bar{N}$} & \multicolumn{6}{|c|}{ Statistically equivalent area fraction $\bar{A}_{\mathrm{fp}}$} \\
\hline & \multicolumn{6}{|c|}{ Volume fraction } & \multicolumn{6}{|c|}{ Volume fraction } \\
\hline & 0.05 & 0.10 & 0.15 & 0.20 & 0.2209 & 0.25 & 0.05 & 0.10 & 0.15 & 0.20 & 0.2209 & 0.25 \\
\hline 1 & 0.84 & 1.00 & 1.00 & 1.00 & 1.00 & 1.00 & 0.0925 & 0.2134 & 0.3110 & 0.3938 & 0.4255 & 0.4672 \\
\hline 2 & 1.08 & 1.55 & 2.00 & 2.00 & 2.00 & $\mathrm{n} / \mathrm{a}$ & 0.0738 & 0.1561 & 0.2483 & 0.3306 & 0.3604 & $\mathrm{n} / \mathrm{a}$ \\
\hline 3 & 1.31 & 1.74 & 2.12 & 3.00 & 3.00 & 3.00 & 0.0684 & 0.1380 & 0.1989 & 0.2650 & 0.2944 & 0.3269 \\
\hline 4 & 1.52 & 1.95 & 2.24 & 2.58 & 2.84 & 3.57 & 0.0659 & 0.1299 & 0.1840 & 0.2245 & 0.2427 & 0.2783 \\
\hline 5 & 1.73 & 2.16 & 2.42 & 2.56 & 2.65 & 2.85 & 0.0647 & 0.1251 & 0.1742 & 0.2044 & 0.2121 & 0.2231 \\
\hline 6 & 1.92 & 2.38 & 2.60 & 2.69 & 2.71 & 2.77 & 0.0639 & 0.1232 & 0.1693 & 0.1983 & 0.2012 & 0.2040 \\
\hline 7 & 2.10 & 2.61 & 2.81 & 2.85 & 2.80 & 2.73 & 0.0630 & 0.1230 & 0.1677 & 0.1956 & 0.1962 & 0.1872 \\
\hline 8 & 2.28 & 2.83 & 3.02 & 3.05 & 2.98 & 2.80 & 0.0626 & 0.1230 & 0.1674 & 0.1987 & 0.2025 & 0.1927 \\
\hline 9 & 2.45 & 3.04 & 3.25 & 3.29 & 3.24 & 2.97 & 0.0618 & 0.1223 & 0.1691 & 0.2072 & 0.2191 & 0.2141 \\
\hline 10 & 2.60 & 3.26 & 3.50 & 3.57 & 3.58 & 3.48 & 0.0616 & 0.1225 & 0.1715 & 0.2140 & 0.2343 & 0.2541 \\
\hline 12 & 2.91 & 3.67 & 3.98 & 4.12 & 4.17 & 4.23 & 0.0608 & 0.1219 & 0.1747 & 0.2228 & 0.2475 & 0.2836 \\
\hline 14 & 3.20 & 4.03 & 4.45 & 4.64 & 4.71 & 4.80 & 0.0602 & 0.1206 & 0.1769 & 0.2278 & 0.2532 & 0.2927 \\
\hline 16 & 3.46 & 4.39 & 4.90 & 5.14 & 5.24 & 5.34 & 0.0595 & 0.1204 & 0.1780 & 0.2312 & 0.2569 & 0.2975 \\
\hline 18 & 3.73 & 4.74 & 5.33 & 5.64 & 5.76 & 5.89 & 0.0594 & 0.1199 & 0.1782 & 0.2339 & 0.2598 & 0.3015 \\
\hline 20 & 3.96 & 5.05 & 5.72 & 6.12 & 6.27 & 6.46 & 0.0589 & 0.1191 & 0.1777 & 0.2354 & 0.2609 & 0.3038 \\
\hline 24 & 4.44 & 5.64 & 6.42 & 6.99 & 7.21 & 7.55 & 0.0584 & 0.1178 & 0.1759 & 0.2352 & 0.2603 & 0.2993 \\
\hline 32 & 5.29 & 6.74 & 7.67 & 8.43 & 8.71 & 9.11 & 0.0574 & 0.1162 & 0.1729 & 0.2303 & 0.2542 & 0.2815 \\
\hline 48 & 6.82 & 8.68 & 9.87 & 10.76 & 11.07 & 11.38 & 0.0565 & 0.1140 & 0.1696 & 0.2236 & 0.2458 & 0.2726 \\
\hline 64 & 8.16 & 10.37 & 11.82 & 12.86 & 13.21 & 13.65 & 0.0558 & 0.1125 & 0.1680 & 0.2216 & 0.2427 & 0.2730 \\
\hline 100 & 10.82 & 13.75 & 15.69 & 17.14 & 17.61 & 18.25 & 0.0549 & 0.1109 & 0.1659 & 0.2192 & 0.2410 & 0.2712 \\
\hline 200 & 16.83 & 21.37 & 24.47 & 26.75 & 27.52 & 28.59 & 0.0539 & 0.1086 & 0.1627 & 0.2156 & 0.2370 & 0.2672 \\
\hline 400 & 26.32 & 33.39 & 38.21 & 41.84 & 43.16 & 44.79 & 0.0531 & 0.1068 & 0.1602 & 0.2125 & 0.2342 & 0.2638 \\
\hline 1000 & 47.71 & 60.47 & 69.21 & 75.96 & 79.37 & 81.46 & 0.0522 & 0.1050 & 0.1575 & 0.2093 & 0.2338 & 0.2606 \\
\hline
\end{tabular}

a small positive value to simplify the mesh generation.

For the 2D simulations, we introduce two modeling approaches: (i) generation of the models based on intersecting the $3 \mathrm{D}$ specimen with the $X Y$-plane and (ii) generation of models containing same-size circular inclusions employing the statistically equivalent area fraction (see Sec. 3).

As an example of mesh sizes in this work, approximate and representative mesh sizes are as follows: For 3D meshes: aligned meshes typically contain 100,000 nodes $(300,000$ DOF), and unaligned meshes 360,000 nodes $\left(1.08 \times 10^{6} \mathrm{DOF}\right)$. For $2 \mathrm{D}$ meshes: typical meshes using the direct cutting approach have 48,000 nodes $(144,000 \mathrm{DOF})$ and using the equivalent area fraction 30,000 nodes (90,000 DOF). The CPU computational time will depend directly on the platform, but as noted previously the computational time is approximately proportional to $\mathrm{DOF}^{3}$. Consequently, the $3 \mathrm{D}$ samples are considerably more expensive, approximately a factor of 1000 .

In all investigated cases, the specimens consist of an aluminum matrix reinforced with boron spherical particles. The material properties of the two constituents are taken from Ref. [6] and are presented in Table 2.

To ensure that the routine was properly incorporated, we verified (for both 2D- and 3D-FE models) the implementation by considering the entire specimen domain where the matrix and the reinforcement have the same material properties (see Table 2). Thus, the input bulk and shear moduli were $K_{\text {input }}=77.9 \mathrm{GPa}$ and $G_{\text {input }}=24.9 \mathrm{GPa}$, respectively. The averaging relations (6) (implemented as a computational postprocessing procedure) were used to compute the output effective material properties. The accuracy is very good, with relative errors between the input moduli and output effective moduli of order of $10^{-4} \%$ for the bulk modulus and of $10^{-5} \%$ for the shear modulus.

4.2 Effective Properties Based on 2D-Finite Element Models. In this section, we present the effective elastic properties (i.e., bulk and shear moduli) obtained using 2D-FE models. In all 2D cases, plane strain elements are used. The effective properties are estimated using the computational scheme described in Sec. 3 for the volume fractions: $0.05,0.10,0.15,0.20,0.2209$, and 0.25 . For each of the volume fraction studied, 50 random configurations

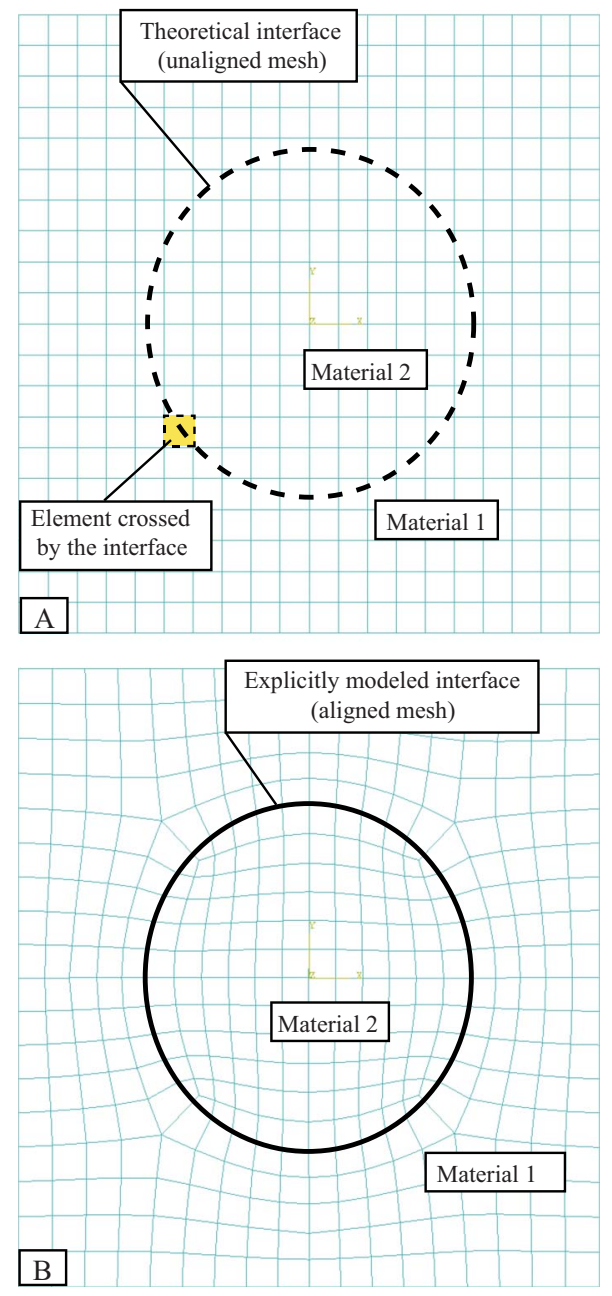

Fig. 6 Modeling inclusions using (a) unaligned mesh and (b) aligned mesh 
Table 2 Material properties of the matrix and particles

\begin{tabular}{lccc}
\hline \hline Constituent & Material & $\begin{array}{c}\text { Bulk modulus } \\
(\mathrm{GPa})\end{array}$ & $\begin{array}{c}\text { Shear modulus } \\
(\mathrm{GPa})\end{array}$ \\
\hline Matrix & Aluminum (Al) & 77.9 & 24.9 \\
Particles & Boron (B) & 230 & 172 \\
\hline \hline
\end{tabular}

are considered.

Two approaches were used, according to the following.

(i) Mesh based on cross-sectioning the 3D specimens with the plane $X Y \quad(z=0)$ : A valid 3D-RVE containing 100 spherical particles is first generated using the algorithm described in Sec. 2 (Fig. 7(a)). The 3D particle configuration is cross-sectioned at the $X Y$ plane $(z=0)$ and the information describing the intersections (i.e., $2 \mathrm{D}$ circular regions) is used to automatically generate the FE model (Fig. 7(b)). This modeling approach results in 2D-FE models containing circular particles of various diameters as visualized in Fig. 7(b).

(ii) Mesh containing same-size circular particles using the statistically equivalent area fraction $\bar{A}_{\mathrm{fp}}$, and the average number of intersected particles: 2D-FE models containing circular particles with a constant diameter are generated. For each investigated volume fraction, the $\bar{A}_{\mathrm{fp}}$ (Sec. 2) was selected to correspond to 100 spherical particles (Fig. 4). Similarly, the mean number of intersected particles was selected for the same volume fraction and the same number of spherical particles (Fig. 5). Based on the obtained value, the closest integer was selected as the radius of the particles. ${ }^{3}$ The circular particles were inserted using the approach described for 3D specimens in Sec. 2.

For the automatic generation of the 2D-FE models we took advantage of a developed modeling frame presented elsewhere [32].

The effective bulk and shear moduli obtained using the two 2D-FE modeling approaches are compared with the HashinShtrikman bounds [7] and the prediction given by the selfconsistent method [8] in Fig. 8. (The Hashin-Shtrikman bounds and the self-consistent method are summarized in the Appendix for completeness of the presentation.) The two 2D-FE modeling approaches produced very close means of the effective bulk and shear moduli. Thus, the major influence on the effective properties is the statistically equivalent area fraction and not the actual distribution of particle sizes.

However, a significant difference is noticed in the standard deviation (indicated in Fig. 8 by the vertical bars). The mesh based on cross-sectioning a 3D configuration, as shown Fig. 7(b), results in a higher standard deviation, which is driven by the variation in the particular area fraction for a specific 2D model. When the statistically equivalent area fraction is employed for 2D models (with same-size particles, Fig. 7(c)), the standard deviation is small since it is only influenced by the random locations of the particles.

4.3 Effective Properties Based on 3D-Finite Element Models. Both aligned and unaligned meshes are considered for the 3D-finite element models (Fig. 9). The random specimens used for the 3D-FE models contained 32 particles completely em-

${ }^{3}$ For example, for a 3D sample containing 100 spherical particles at $25 \%$ volume fraction, the statistically equivalent area fraction is 0.271 and the mean of the intersected number of particles is 18.2 (Table 1). Therefore, the 2D-FE model will contain 18 circular inclusions of the same-size accounting for $27.1 \%$ of the sample area Thus, the radius of the inclusions is the radius of one inclusion representing $27.1 \%$ of a sample of unit area. The size of the $2 \mathrm{D}$ rectangular sample is calculated to accommodate 18 circular inclusions representing $27.1 \%$ of the sample area.
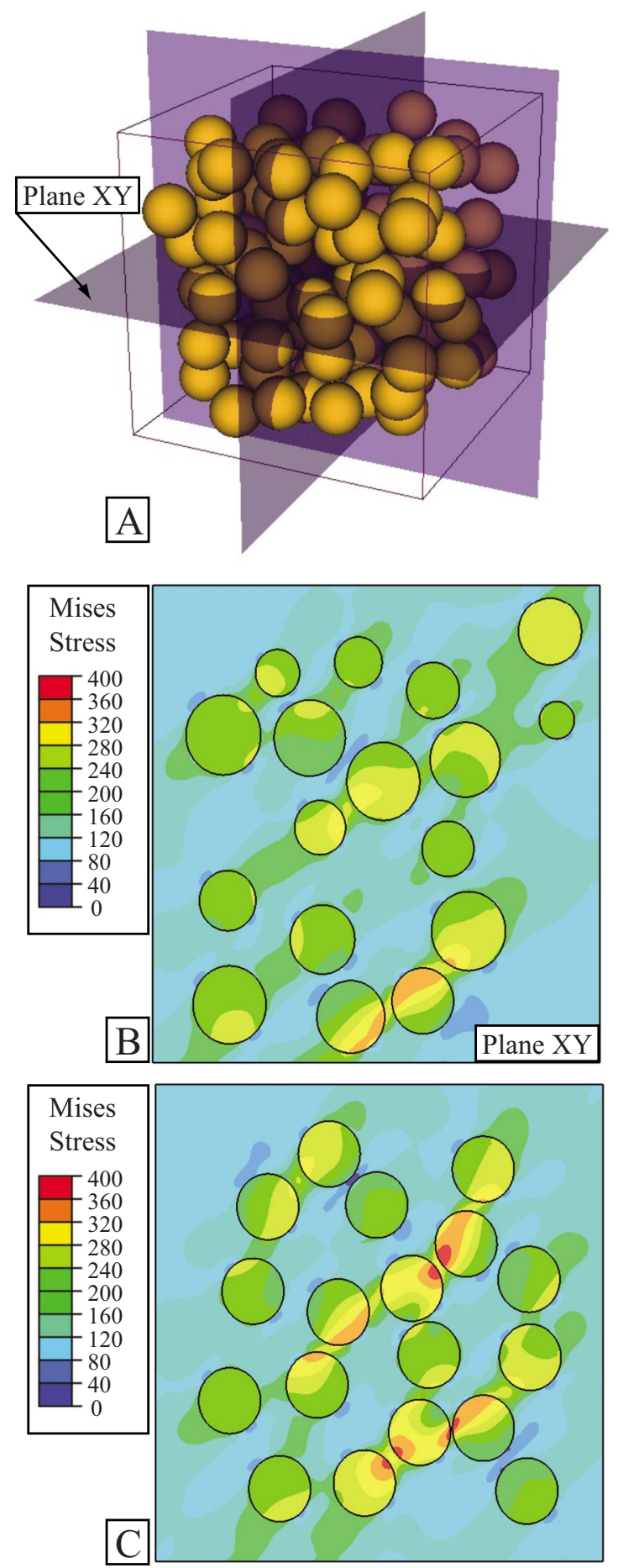

Fig. 7 Two-dimensional-FE modeling approaches: (a) a 3D configuration containing 100 randomly distributed spherical particles $\left(V_{f}=0.25\right),(b)$ the 2D-FE model obtained by crosssectioning the $3 \mathrm{D}$ specimen with the plane $X Y$, and $(c)$ the equivalent 2D model containing 18 same-size circular particles $\left(\boldsymbol{A}_{f}=0.271\right)$

bedded in the matrix (Fig. 9). Investigating 3D-FE models with more particles was found to be computationally prohibitive with our current computational resources.

The effective properties obtained by the two 3D-modeling approaches are presented in Fig. 10. In this figure, the FE results are compared with the Hashin-Shtrikman bounds and the estimations by the self-consistent method. For the aligned meshes, the effective bulk modulus is very close to the lower Hashin-Shtrikman 

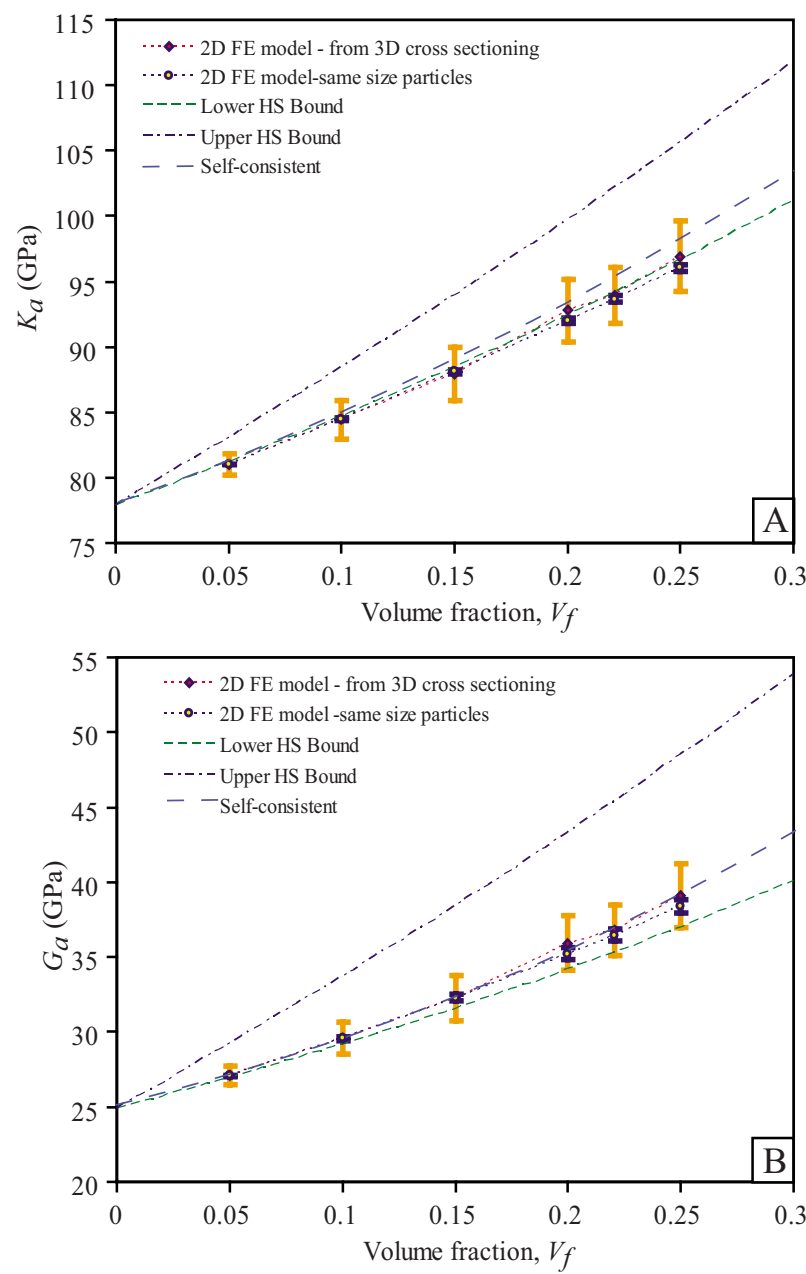

Fig. 8 Effective elastic properties as a function of volume fraction $V_{f}$ predicted by the 2D-FE models using the two modeling approaches, compared with Hashin-Shtrikman bounds and the self-consistent method: (a) the bulk modulus $K_{a}$ and $(b)$ the shear modulus $G_{a}$. The vertical bars indicate \pm 1 standard deviation.

bound, whereas the effective shear modulus is close to the estimate given by the self-consistent method. The effective properties obtained by using unaligned meshes are consistently higher than for those resulting from the self-consistent method (Fig. 10). The standard deviation was about 1.5 times higher for the unaligned meshes than the aligned meshes.

4.4 Discussion. The 2D numerical approaches both give similar mean values of the effective bulk and shear moduli as the $3 \mathrm{D}$ approaches (Fig. 11). The effective bulk modulus (i.e., the mean based on 50 specimens) is very close to the lower HashinShtrikman bound. The effective shear modulus is very close to the estimate given by the self-consistent method. However, the results from the $2 \mathrm{D}$ models based on cross-sectioning a 3D configuration, have significantly larger standard deviations.

An additional comparison can be made to work presented by Zohdi and Wriggers [6]. They considered the volume fraction $22.09 \%$ for unaligned 3D-FE meshes only. The effective properties obtained in that work is included in Table 3 (unaligned meshes with $24 \times 24 \times 24$ elements representing specimens with 20 particles [6]). The comparison suggests that the unaligned mesh overestimate the shear modulus and underestimate the bulk modulus.

Although 3D models are preferred for evaluating the effective properties distribution, the comparisons suggest that the mean val-
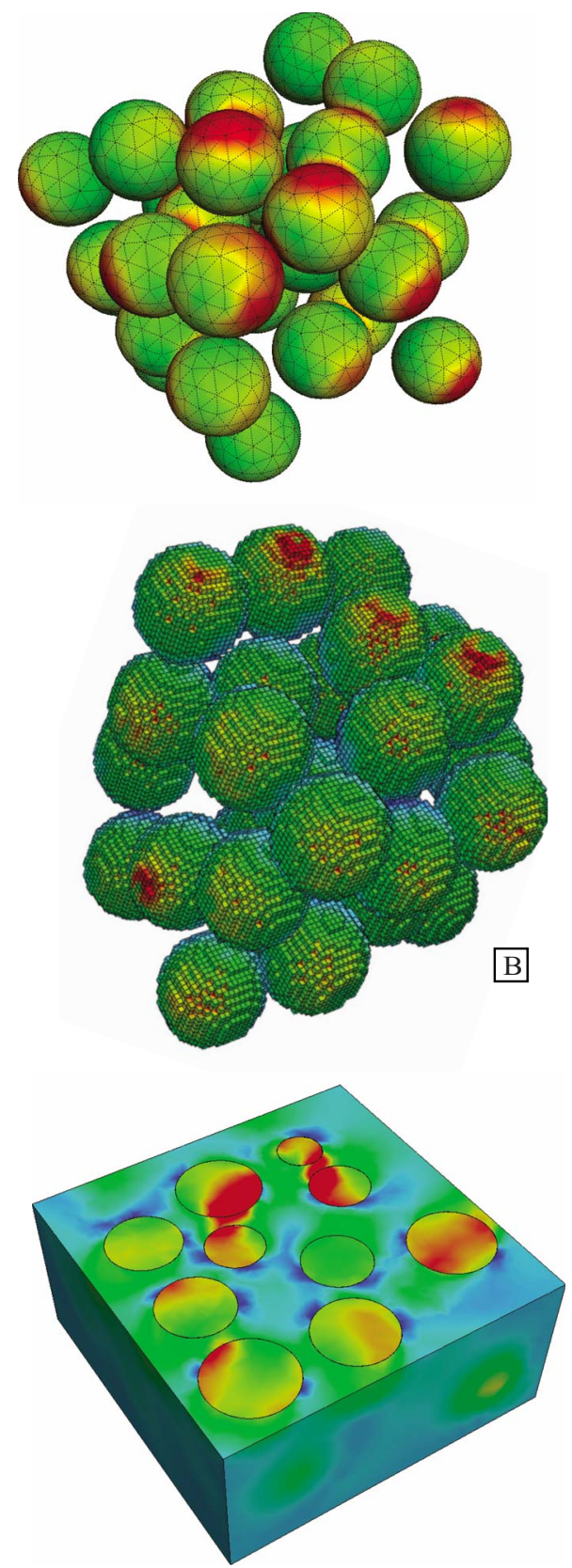

Fig. 9 Three-dimensional-FE models of RVEs containing random configurations of 32 particles at $25 \%$ volume fraction: (a) aligned tetrahedral mesh, $(b)$ unaligned hexahedral mesh, and (c) cross section along the plane $X Y$. The contour plots show Mises stress

ues provided by the $2 \mathrm{D}$ models very closely reproduced the results obtained from the 3D models with aligned mesh of quadratic tetrahedral elements. Even though 3D models may be more realistic, they frequently become too large to use in most practical applications. In these cases, 2D models based on the statistically equivalent area fraction $\bar{A}_{\text {fp }}$ can be successfully used to estimate the effective properties. To generate a suitable model, our results presented in Table 1 can be used to determine the statistically equivalent area fraction $\bar{A}_{\mathrm{fp}}$ and the number of intersected particles $\bar{N}$, for a given volume fraction.

In future studies, we will investigate if other microstructures and microstructural features (such as flaws) can be investigated using the concept of statistically equivalent area fraction. 

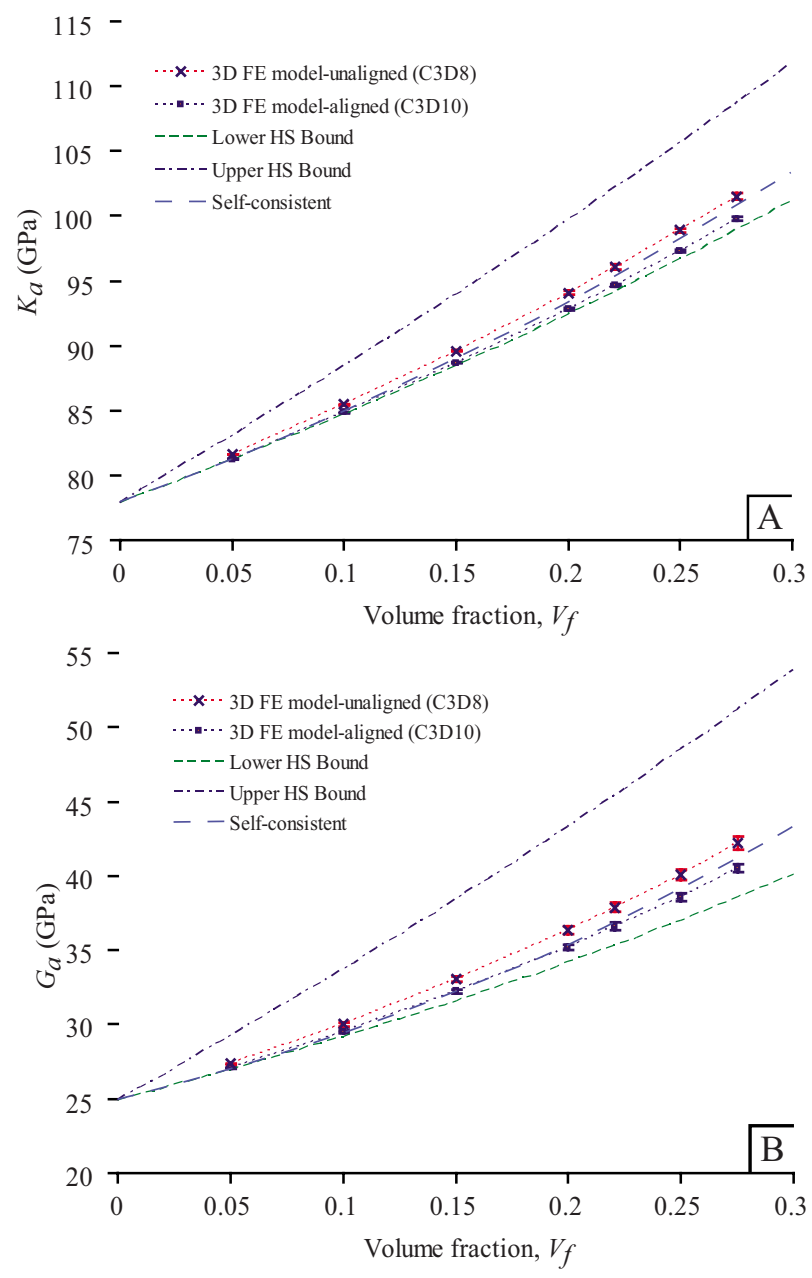

Fig. 10 Effective elastic properties as a function of the volume fraction $V_{f}$ predicted by the 3D-FE models using aligned and unaligned meshes compared with Hashin-Shtrikman bounds and the self-consistent method: (a) bulk modulus $K_{a}$ and (b) shear modulus $G_{a}$

\section{Concluding Remarks}

A FEM based computational procedure for estimating the effective elastic properties of a particle reinforced matrix has been investigated. The method is applied to a macroscopically isotropic medium reinforced with randomly distributed spherical particles of uniform diameter, aiming to establish the overall elastic response. Of particular interest in this study was to investigate if it is possible to obtain the correct effective bulk and shear moduli by representing the $3 \mathrm{D}$ structure in a $2 \mathrm{D}$ configuration.

To this end, a computational scheme was adopted to enable both 2D- and 3D-finite element models using particle volume fractions in the range of $0.05-0.275$. Since the particles are randomly distributed, it is not possible to indentify a representative volume element with only one particle. Thus, the effective properties were averaged using 50 specimens for all cases considered. For the 2D models, we used aligned meshes only, whereas for 3D models we used both aligned and unaligned meshes. A RSA based Monte-Carlo simulation was initially conducted to establish the proposed statistically equivalent area fraction and the average number of intersected particles. The statistically equivalent area fraction defined in this work depends on two parameters: the volume fraction of the 3D specimen and the number of particles in the $3 \mathrm{D}$ specimen. The volume fraction investigated here is limited to lower volume fractions due to that the selected method for

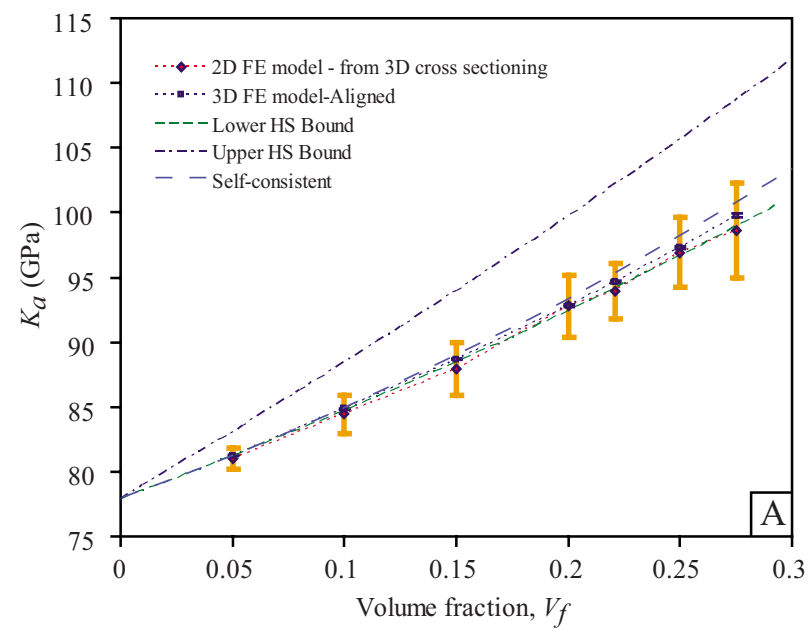

$55-$
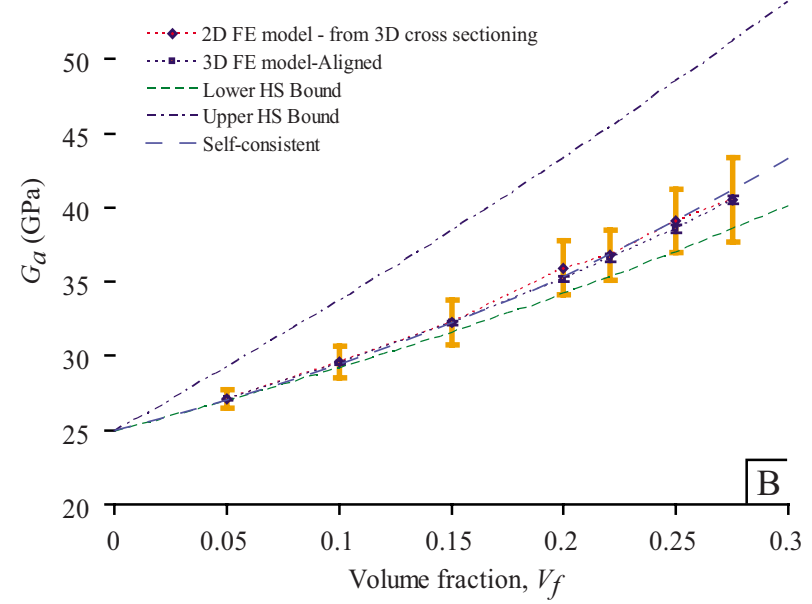

Fig. 11 Effective properties as a function of the volume fraction $V_{f}$ predicted by 2D- (direct generation) and 3D-FE models with aligned meshes: (a) bulk modulus $K_{a}$ and $(b)$ shear modulus $G_{a}$. The vertical bars indicate \pm 1 standard deviation.

randomly generating particles is bounded to this volume fraction. However, the concept of statistically equivalent area fraction is not limited to this low volume fraction only.

When the computational procedure was used with 2D-FE models, we found that the distribution of particle sizes has only a minor influence on the effective properties. However, the statistically equivalent area fraction directly affects the values of the effective properties.

Using the aligned meshes, the 2D-FE models gave very close estimates of the mean of the effective properties to that of the 3D-FE models. Therefore, when considering a matrix reinforced with randomly distributed spherical particles, a 2D modeling approach can be used to estimate the mean of the effective properties at a reduced computational cost. We introduced two possible methods to create a representative modeling scheme: (i) a mesh generated by cross-sectioning the $3 \mathrm{D}$ specimens with plane $X Y$ and (ii) a mesh generated by using the statistically equivalent area fraction with same-size circular particles. The data for the latter case was generated via the approach presented in Sec. 3, which results in the data presented in Table 1.

Thus, using the statistically equivalent area fraction along with the mean number of intersected particles, introduced in this work, may be the most efficient way to estimate effective elastic properties. These values are presented in Table 1 and can directly be used for future investigations. Ongoing studies are utilizing this 


\begin{tabular}{|c|c|c|c|}
\hline Case & $\begin{array}{c}K_{a} \\
(\mathrm{GPa})\end{array}$ & $\begin{array}{c}G_{a} \\
(\mathrm{GPa})\end{array}$ & Comments \\
\hline \multicolumn{4}{|l|}{ Zohdi and Wriggers [6] using an unaligned mesh with } \\
\hline $24 \times 24 \times 24$ elements & 96.17 & 42.35 & \multirow{2}{*}{$\begin{array}{l}\text { Trilinear hexahedral elements } \\
\text { 2D particles generated directly by cross-sectioning the } \\
\text { 3D configuration }\end{array}$} \\
\hline 2D-FE-direct generation (aligned mesh) & 93.93 & 36.78 & \\
\hline \multicolumn{4}{|l|}{$2 \mathrm{D}-\mathrm{FE}$ - using the equivalent area fraction (aligned } \\
\hline mesh) & 93.65 & 36.47 & 2D particles of the same size \\
\hline 3D-FE-unaligned mesh with $60 \times 60 \times 60$ elements & 96.24 & 38.06 & Trilinear hexahedral elements (C3D8) \\
\hline $3 \mathrm{D}-\mathrm{FE}$ - unaligned mesh with $70 \times 70 \times 70$ elements & 96.04 & 37.87 & Trilinear hexahedral elements (C3D8) \\
\hline 3D-FE_-aligned mesh & 94.64 & 36.61 & Quadratic tetrahedral elements (C3D10) \\
\hline Self-consistent method & 95.28 & 36.86 & Analytical \\
\hline
\end{tabular}

concept for investigating more sophisticated composite structures, such as coated particles and damaged structures, with nonelastic properties.

\section{Appendix: Analytical Methods}

The HS bounds [7] are the tightest bounds that can be derived without taking into consideration the geometry of the microstructure. They predict that the effective bulk and shear moduli should be bounded by $K_{\mathrm{HS}}^{-} \leq K_{a} \leq K_{\mathrm{HS}}^{+}$and $G_{\mathrm{HS}}^{-} \leq G_{a} \leq G_{\mathrm{HS}}^{+}$, respectively, where

$K_{\mathrm{HS}}^{-}$is the lower HS bound on the bulk modulus

$$
K_{\mathrm{HS}}^{-}=K_{m}+\frac{V_{\mathrm{fp}}}{\frac{1}{K_{p}-K_{m}}+\frac{3 V_{\mathrm{fm}}}{3 K_{m}+4 G_{m}}}
$$

$K_{\mathrm{HS}}^{+}$is the upper HS bound on the bulk modulus

$$
K_{\mathrm{HS}}^{+}=K_{p}+\frac{V_{\mathrm{fm}}}{\frac{1}{K_{m}-K_{p}}+\frac{3 V_{\mathrm{fp}}}{3 K_{p}+4 G_{p}}}
$$

$G_{\mathrm{HS}}^{-}$is the lower HS bound on the shear modulus

$$
G_{\mathrm{HS}}^{-}=G_{m}+\frac{V_{\mathrm{fp}}}{\frac{1}{G_{p}-G_{m}}+\frac{6 V_{\mathrm{fm}}\left(K_{m}+2 G_{m}\right)}{5 G_{m}\left(3 K_{m}+4 G_{m}\right)}}
$$

$G_{\mathrm{HS}}^{+}$is the upper HS bound on the shear modulus

$$
G_{\mathrm{HS}}^{+}=G_{p}+\frac{V_{\mathrm{fm}}}{\frac{1}{G_{m}-G_{p}}+\frac{6 V_{\mathrm{fp}}\left(K_{p}+2 G_{p}\right)}{5 G_{p}\left(3 K_{p}+4 G_{p}\right)}}
$$

In the relations above, $K_{m}, G_{m}$ represent the elastic properties of the matrix and $K_{p}, G_{p}$ of the particles, $V_{\mathrm{fp}}$ is the particle volume fraction, and $V_{\mathrm{fm}}=1-V_{\mathrm{fp}}$ is the matrix volume fraction.

The self-consistent method [8], applied to media reinforced with spherical particles, assumes that the effective bulk and shear moduli are the solution of the following system of coupled algebraic equations [2]:

$$
\begin{gathered}
\frac{K_{a}}{K_{m}}=1-V_{\mathrm{fp}} \frac{K_{a}\left(K_{m}-K_{p}\right)}{K_{m}\left(K_{a}-K_{p}\right)} \frac{K_{a}}{K_{a}-K_{p}}-\frac{1+\nu_{a}}{3\left(1-\nu_{a}\right)} \\
\frac{G_{a}}{G_{m}}=1-V_{\mathrm{fp}} \frac{G_{a}\left(G_{m}-G_{p}\right)}{G_{m}\left(G_{a}-G_{p}\right)} \frac{G_{a}}{G_{a}-G_{p}}-\frac{2\left(4-5 \nu_{a}\right)}{15\left(1-\nu_{a}\right)}
\end{gathered}
$$

where $\nu_{a}=\left(3 K_{a}-2 G_{a}\right) /\left(6 K_{a}+2 G_{a}\right)$ is the effective Poisson ratio. The solution for $K_{a}, G_{a}$ is obtained using an iterative numerical procedure.

\section{References}

[1] Temizer, I., and Zohdi, T. I., 2007, "A Numerical Method for Homogenization in Non-Linear Elasticity," Comput. Mech., 40, pp. 281-298.

[2] Nemat-Nasser, S., and Hori, M., 1999, Micromechanics: Overall Properties of Heterogeneous Materials, Elsevier, New York.

[3] Qu, J., and Cherkaoui, M., 2006, Fundamentals of Micromechanics of Solids, Wiley, New York.

[4] Torquato, S., 2001, Random Heterogeneous Materials, Springer, New York.

[5] Zohdi, T. I., and Wriggers, P., 2005, Introduction to Computational Mechanics, Springer-Verlag, Berlin.

[6] Zohdi, T. I., and Wriggers, P., 2001, "Aspects of the Computational Testing of the Mechanical Properties of Microheterogeneous Material Samples," Int. J. Numer. Methods Eng., 50, pp. 2573-2599.

[7] Hashin, Z., and Shtrikman, S., 1963, "A Variational Approach to the Theory of the Elastic Behaviour of Multiphase Materials," J. Mech. Phys. Solids, 11, pp. $127-140$.

[8] Hill, R., 1965, "A Self-Consistent Mechanics of Composite Materials," J. Mech. Phys. Solids, 13, pp. 213-222.

[9] Ishai, O., and Cohen, L. J., 1967, "Elastic Properties of Filled and Porous Epoxy Composites," Int. J. Mech. Sci., 9, pp. 539-546.

[10] Kenyon, A. S., and Duffey, H. J., 1967, "Properties of a Particulate-Filled Polymer," Polym. Eng. Sci., 7, pp. 189-193.

[11] Smith, J. C., 1976, "Experimental Values for Elastic-Constants of a Particulate-Filled Glassy Polymer," J. Res. Natl. Bur. Stand., Sect. A, 80, pp. 45-49.

[12] Smith, J. C., 1976, "Elastic-Constants of a Particulate-Filled Glassy PolymerComparison of Experimental Values With Theoretical Predictions," Polym. Eng. Sci., 16, pp. 394-399.

[13] Richard, T. G., 1975, "Mechanical-Behavior of a Solid Microsphere Filled Composite," J. Compos Mater., 9, pp. 108-113.

[14] Cipra, B., 1998, "Mathematics-Packing Challenge Mastered at Last," Science, 281, p. 1267.

[15] Jaeger, H. M., and Nagel, S. R., 1992, "Physics of the Granular State," Science, 255, pp. 1523-1531.

[16] Hertz, P., 1909, "Concerning the Average Mutual Distance Between Points, Which Are Represented in Space With a Common Density," Math. Ann., 67, pp. 387-398.

[17] Metropolis, N., Rosenbluth, A. W., Rosenbluth, M. N., Teller, A. H., and Teller, E., 1953, "Equation of State Calculations by Fast Computing Machines," J. Chem. Phys., 21, pp. 1087-1092.

[18] Talbot, J., and Schaaf, P., 1989, "Random Sequential Adsorption of Mixtures," Phys. Rev. A, 40, pp. 422-427.

[19] Tewari, A., and Gokhale, A. M., 2004, "Nearest-Neighbor Distances Between Particles of Finite Size in Three-Dimensional Uniform Random Microstructures," Mater. Sci. Eng., A, 85, pp. 332-341.

[20] Tan, H., Huang, Y., Liu, C., and Geubelle, P. H., 2005, "The Mori-Tanaka Method for Composite Materials With Nonlinear Interface Debonding," Int. J. Plast., 21, pp. 1890-1918.

[21] Rintoul, M. D., and Torquato, S., 1997, "Reconstruction of the Structure of Dispersions," J. Colloid Interface Sci., 186, pp. 467-476.

[22] Segurado, J., and Llorca, J., 2002, "A Numerical Approximation to the Elastic Properties of Sphere-Reinforced Composites," J. Mech. Phys. Solids, 50, pp. 2107-2121.

[23] PYTHON, 2005, “PYTHON Documentation, Release 2.3.5," http:// www.python.org/doc/

[24] Lutz, M., 1996, "Programming PYthon," O’Reilly, Sebastopol, CA.

[25] Matsumoto, M., and Nishimura, T., 1998, "Mersenne Twister: A 623Dimensionally Equidistributed Uniform Pseudorandom Number Generator,' ACM Trans. Model. Comput. Simul., 8, pp. 3-30.

[26] Zohdi, T. I., 2004, "Homogenization Methods and Multiscale Modeling," Encyclopedia of Computational Mechanics, Vol. 2, R. Stein, R. de Borst, and T. J. R. Hughes, eds., Wiley, New York, pp. 407-430. 
[27] Bathe, K.-J., 1996, Finite Element Procedure, Prentice-Hall, Upper Saddle River.

[28] Hughes, T. J. R., 2000, The Finite Element Method: Linear Static and Dynamic Finite Element Analysis, Dover, Mineola, NY.

[29] Kaliakin, V. N., 2002, Introduction to Approximate Solution Techniques, Numerical Modeling and Finite Element Methods, Marcel Dekker, New York.
[30] ABAQUS, 2006, ABAQUS 6.6 User Subroutines Reference Manual, ABAQUS, Inc., Providence, RI.

[31] ABAQUS, 2006, ABAQUS 6.6 Theory Manual, ABAQUS, Inc., Providence, RI.

[32] Cojocaru, D., and Karlsson, A. M., 2008, "An Object-Oriented Approach for Modeling and Simulation of Crack Growth in Cyclically Loaded Structures," Adv. Eng. Software, 39, pp. 995-1009. 